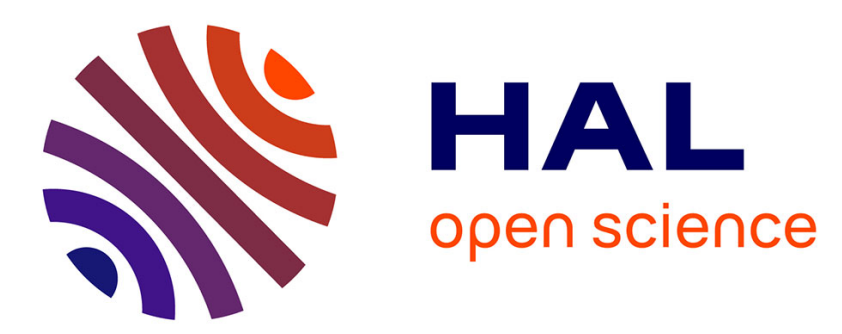

\title{
Accuracy of Lead Removal vs Linear Interpolation in Non-Invasive Electrocardiographic Imaging (ECGI)
}

Laura Bear, Mark Potse, Josselin Duchateau, Nejib Zemzemi, Yves Coudière, Rémi Dubois

\section{- To cite this version:}

Laura Bear, Mark Potse, Josselin Duchateau, Nejib Zemzemi, Yves Coudière, et al.. Accuracy of Lead Removal vs Linear Interpolation in Non-Invasive Electrocardiographic Imaging (ECGI). Computing in cardiology, Sep 2015, Nice, France. hal-01241752

\section{HAL Id: hal-01241752 \\ https://inria.hal.science/hal-01241752}

Submitted on 10 Dec 2015

HAL is a multi-disciplinary open access archive for the deposit and dissemination of scientific research documents, whether they are published or not. The documents may come from teaching and research institutions in France or abroad, or from public or private research centers.
L'archive ouverte pluridisciplinaire HAL, est destinée au dépôt et à la diffusion de documents scientifiques de niveau recherche, publiés ou non, émanant des établissements d'enseignement et de recherche français ou étrangers, des laboratoires publics ou privés. 


\title{
Accuracy of Lead Removal vs Linear Interpolation in Non-Invasive Electrocardiographic Imaging (ECGI)
}

\author{
Laura Bear $^{1,4}$, Mark Potse ${ }^{1,2,3}$, Josselin Duchateau ${ }^{1,4,5}$, Nejib Zemzemi ${ }^{1,2}$, \\ Yves Coudière $^{1,2,3}$, Rémi Dubois ${ }^{1,4}$ \\ ${ }^{1}$ Electrophysiology and Heart Modelling Institute (IHU LIRYC), Bordeaux, France. \\ ${ }^{2}$ Inria Bordeaux Sud-Ouest. Carmen team, Bordeaux, France \\ ${ }^{3}$ IMB UMR CNRS 5251, Bordeaux, France \\ ${ }^{4}$ CRCTB Inserm U1045, Bordeaux, France \\ ${ }^{5}$ Université de Bordeaux, CRCTB Inserm U1045, Bordeaux, France
}

\begin{abstract}
This study examines the effect of missing body surface potentials on inverse solutions, and determines if linear interpolation can regain information lost. Using simulated and experimental data, electrograms (EGMs) were reconstructed after 1) removing and 2) interpolating 'broken' signals. Results showed that losing body surface potentials over the chest can reduce inverse reconstruction accuracy. Linear interpolation did not improve, and could further deteriorate reconstruction accuracy.
\end{abstract}

\section{Introduction}

Non-invasive electrocardiographic imaging (ECGI) of cardiac electrical activity from body surface potentials (BSPs) is a promising clinical tool for electroanatomic mapping of arrhythmogenic substrates [1,2]. Current clinical systems typically oversample BSPs by recording from up to 250 locations across the torso. This allows for redundancy in the recordings, ensuring all information projected from the heart is captured regardless of the cardiac sequence. This also allows for inevitable 'broken' electrodes. That is, signals which are considered unusable due to excessive noise, poor contact, broken wires, etc. Current clinical practice is to reconstruct these missing BSPs using interpolation from the remaining signals $[1,2]$. Whilst it seems intuitive to want to "replenish" these missing signals, it remains unclear whether this is necessary for an accurate inverse solution. An alternative option is to simply remove the missing electrodes from the model, and reconstruct cardiac activity from the remaining signals. Whilst some information from the body surface may be lost, further error will not be introduced into the system through interpolation.

Thus, the aim of this study was first to determine the effect of removing body surface potentials on inverse solutions, and second to determine if linear interpolation can be used to regain any information lost.

\section{Methods}

\subsection{Data Sets}

Numerical Simulations

Simulated data used in this investigation were computed using a monodomain reaction-diffusion model ( $0.2 \mathrm{~mm}$ resolution) coupled to a bidomain torso model ( $1 \mathrm{~mm}$ resolution). Transmembrane ionic currents were computed with the TNNP model of the human ventricular myocyte [3]. Simulations were performed using the propag-5 software [Krause 2011] on a Blue Gene/Q supercomputer. Cardiac sequences were simulated for six patterns of paced activation; five single-stimulus, and one dual-stimulus beat. BSPs were computed from simulated transmembrane currents using a finite-difference torso model. Heart and thoracic anatomy were created from MRI data and included intracavitary blood, anisotropic myocardium, lungs, and an anisotropic skeletal muscle layer. Gaussian noise of $50 \mu \mathrm{V}$ RMS was applied to BSPs (peak-to-peak range of around $0.3 \mathrm{mV}$ ). From the simulated torso potentials, a 252-channel BSP recording was extracted and used here.

\section{Torso-Tank Experiment}

Experimental data came from a human-shaped torso tank, filled with an electrolytic solution and containing an isolated, Langendorff-perfused, canine heart [4]. Tank potentials were recorded from 192 electrodes, simultaneously with epicardial potentials using a 247electrode sock. The LAD of the isolated heart was cannulated and the heart paced from a bipolar hook electrode in the right atrium. Recordings were taken during 4 interventions (20 hearts beats for analysis in total). During two interventions, the pacing rate was 
constant (350 and $325 \mathrm{~ms}$ ) with flow rate through the LAD reduced step-wise from 20 to $0 \mathrm{ml} / \mathrm{min}$. The remaining two interventions had a constant flow rate $(35$ or $25 \mathrm{ml} / \mathrm{min}$ ) with the pacing reduced step-wise from 350 to $275 \mathrm{~ms}$.

\subsection{Problem Formulation}

Epicardial potentials are defined as the underlying source model in a boundary value problem with the Laplace equation [5]. This problem is solved using the method of fundamental solutions [6]. For simplicity, a homogeneous conductivity is assumed within the torso and the inverse solution is found separately at each time instant using Tikhonov regularization, with CRESO criterion to find the regularization parameter.

Unrefined torso meshes were used, with nodes defining electrode locations. For the experimental data, 34 leads were already unusable, situated over the right lateral stomach (see Figure 4). For simulated data, 40 different versions of 'broken' leads were defined: 31 with a single group of 4 to 15 neighboring electrodes, and 9 realistic patterns of 17 to 51 electrodes, directly mapped from clinical data. After defining a subset of electrodes as 'broken', two methods were used to reconstruct epicardial potentials from remaining BSPs: 1) 'Broken' electrodes and associated elements were removed from the torso mesh and excluded from the formulation of the transfer matrix, and 2) a local barycentric interpolation method was used to reconstruct 'broken' potentials. That is, interpolated potentials were defined as the weighted sum of measured potentials from neighbouring electrodes, with weights a function of the Euclidean distance between measured and 'broken' electrodes.
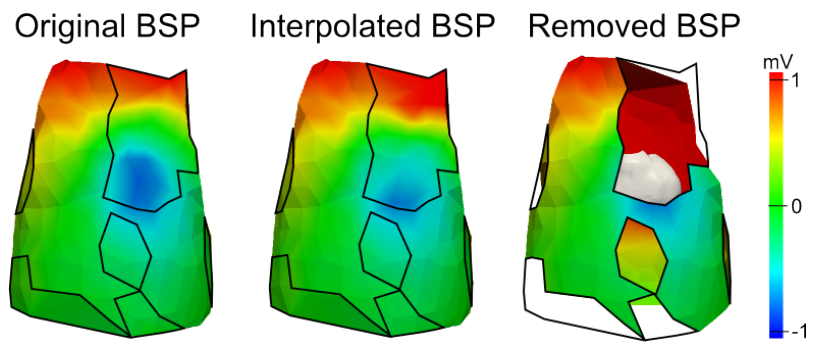

Figure 1. BSP maps $62 \mathrm{~ms}$ into the QRS for a right ventricular pacing sequence. Regions containing a realistic pattern of 'broken' leads are outlined.

Inversely reconstructed EGMs $\left(\emptyset_{S}\right)$ were evaluated relative to 'gold standard' potentials $\left(\emptyset_{M}\right)$; both through visual examination of EGMs, and using relative error norm (RE), and correlation coefficient over time $(\mathrm{t})$ defined as:

$$
\begin{gathered}
R E=\left\|\emptyset_{M}-\emptyset_{S}\right\|_{2} /\left\|\emptyset_{M}\right\|_{2} \\
C C=\frac{\sum_{t}\left(\emptyset_{M}-\mu_{M}\right)\left(\emptyset_{S}-\mu_{S}\right)}{\left\|\emptyset_{M}-\mu_{M}\right\|_{2}\left\|\emptyset_{S}-\mu_{S}\right\|_{2}}
\end{gathered}
$$

For simulated data, 'gold standard' EGMs were those inversely reconstructed from the full set of torso signals. For experimental data, the 'gold standard' was directly recorded EGMs. Normality was tested with a ShapiroWilk's test ( $p>0.05)$. To determine differences, a paired ttest was used for normal data, and a two-sided Wilcoxon signed rank test for non-normal. Statistical significance was accepted for $\mathrm{p}<0.05$.

\section{Results}

\subsection{Numerical Simulations}

Figure 1 presents a BSP map simulated from a cardiac activation sequence with pacing from the anterior $R V$, $62 \mathrm{~ms}$ into the QRS (left). Black lines outline the regions containing a realistic pattern of 43 'broken' leads. BSPs at these electrodes were interpolated from remaining signals (middle), or were removed from the torso mesh (right).

Here we see that the interpolation scheme produced torso potentials that were relatively inaccurate in terms of potential magnitude within the region of high potential gradient over the upper left torso. This is reflected with a high $\mathrm{RE}$ of 0.54 within this region, though a $\mathrm{CC}$ of 0.95 suggests overall the shape of the ECGs was accurate. Removing 'broken' electrodes removed a large portion of the potential minimum centered over the heart (shown in white).
A

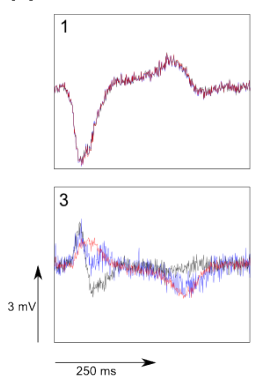

B Removed
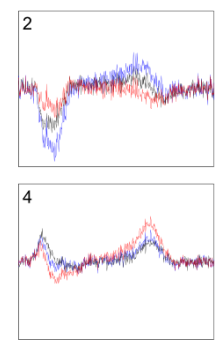

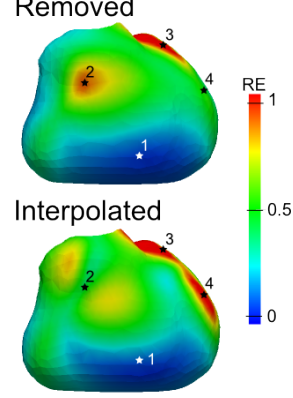

Figure 2. A Representative 'gold standard' EGMs (black) and those reconstructed after removing (blue) and interpolating (red) BSPs, located at black stars in B, showing RE between EGMs on epicardial meshes.

EGMs were reconstructed for each version of BSPs, with representative EGMs presented in Figure 1A. RE values were computed between 'gold standard' EGMs (black) and those reconstructed after removing (blue) and interpolating (red) BSPs respectively, presented in 1B on an anterior view of the epicardium.

Most EGMs were reconstructed faithfully compared to the gold standard, both after removing and interpolating BSPs (e.g. Fig.2, EGM 1). A small region of EGMs reconstructed after removing BSPs showed poor correspondence in potential magnitude on the anterior 
$\mathrm{RV}$, and in a slightly larger region at the base of the LV (e.g. Fig.2, EGMs 2-4), corresponding to high RE values in these regions. Despite this, the topology of these EGMs typically resembled that seen by the gold standard. Interpolating BSP potentials resulted in similar regions of poor correspondence in potential magnitude reconstruction, though these were more dispersed than with removing electrodes. Unlike removing BSPs, in some locations EGMs were completely inverted compared to the 'gold standard'.
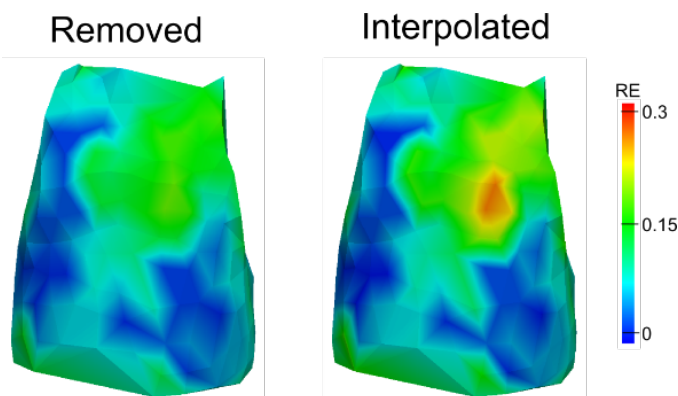

Figure 3. RE between 'gold standard' and reconstructed BSPs, averaged and attributed to the 'broken' electrodes.

Across the data set, the majority of EGMs were reconstructed faithfully when 'broken' BSPs were removed, with $98 \%$ of EGMs having $\mathrm{CC}>0.95$ and $84 \%$ having $\mathrm{RE}<10 \%$. Further inspection revealed that for certain versions of 'broken' leads, small regions on the epicardial surface were poorly reconstructed $(0.02 \%$ of all EGMs had $\mathrm{CC}<0.6$ and $0.02 \%$ had $\mathrm{RE}>100 \%$ ). The mean $\mathrm{RE}$ across the heart was calculated and attributed to the 'broken' electrodes themselves. The average of these values for each cardiac sequence is plotted in Figure 3. Unsurprisingly, the region of largest average RE was situated directly over the heart, suggesting that removing electrodes in this area produces the most substantial changes in reconstructed EGMs. As with removing the BSPs, most EGMs reconstructed with interpolated BSPs were accurate $(97 \%$ with $\mathrm{CC}>0.95 ; 82 \%$ with $\mathrm{RE}<10 \%)$. Interestingly, interpolating to 'broken' leads more frequently resulted in accurate EGMs than removing them $(0.22 \%$ with $\mathrm{CC}<0.6 ; 0.12 \%$ with $\mathrm{RE}>100 \%)$. This difference is statistically significant, as across the data set as a whole $\mathrm{CC}$ are higher, and $\mathrm{RE}$ are lower for removing BSPs then for interpolating $(p=0)$. Again, as with removing electrodes, the highest source of error came from interpolating to electrodes directly over the heart, typically where potentials gradients on the torso are highest.

\subsection{Torso-Tank Experiment}

Figure 4A shows recorded and interpolated tank potential maps at $32 \mathrm{~ms}$ in the QRS during RA pacing every $325 \mathrm{~ms}$, with an LAD flow rate of $35 \mathrm{ml} / \mathrm{min}$. The region with no electrical data from 34 electrodes is highlighted in grey on the measured BSP map. Thus the accuracy of interpolation cannot be evaluated. Directly recorded epicardial potentials provided the "gold standard" for evaluating inverse solutions. EGMs reconstructed from measured (blue) and interpolated (red) BSPs are compared to the 'gold standard' (black) in 4A. $\mathrm{An}$ anterior view of $\mathrm{RE}$ values across the epicardium is shown in $4 \mathrm{~B}$.

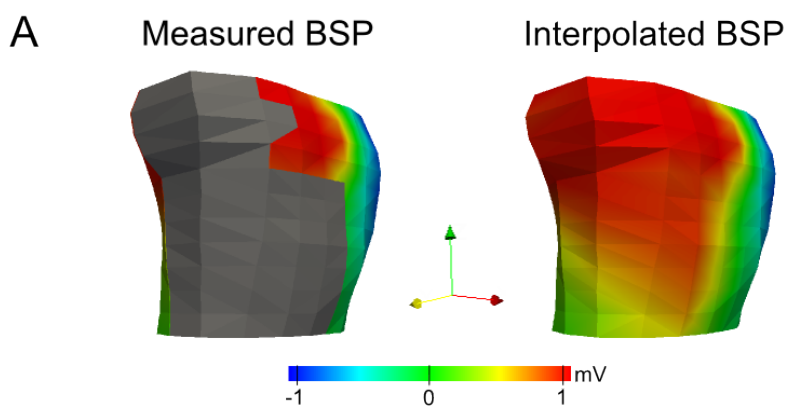

B
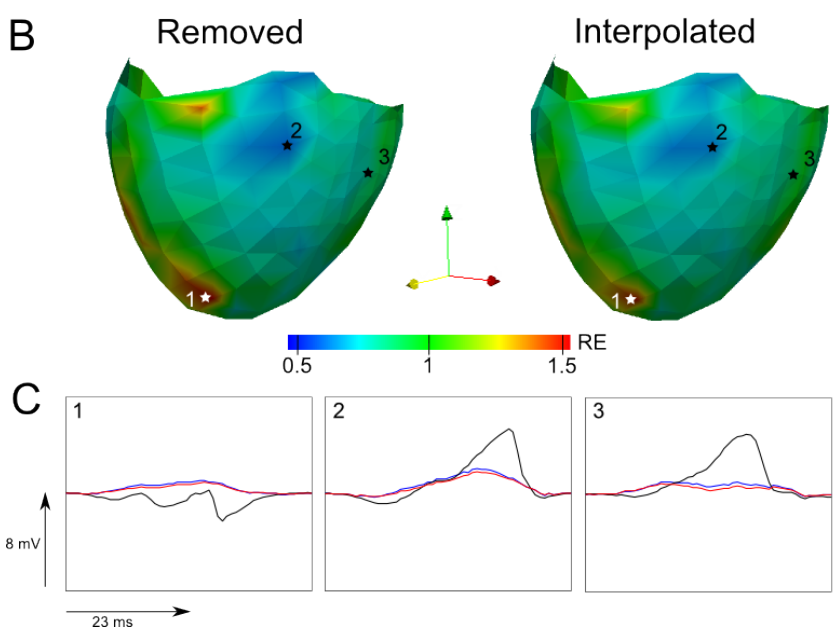

Figure 4. A Measured and interpolated BSP maps $62 \mathrm{~ms}$ into the QRS for a right atrial pacing sequence. Broken electrodes are identified by grey region. B Anterior views of RE values between 'gold standard' EGMs (black) and those reconstructed after removing (blue) and interpolating (red) broken electrodes, with representative EGMs (C) identified by black stars.

Table 1. CC and RE values for torso tank data presented as median [lower quartile;upper quartile]

\begin{tabular}{l|c|c}
\hline & $\mathbf{C C}$ & $\mathbf{R E}$ \\
\hline $\begin{array}{l}\text { Removing } \\
\text { BSPs }\end{array}$ & $0.63[0.44 ; 0.77]$ & $0.86[0.77 ; 0.95]$ \\
\hline $\begin{array}{l}\text { Interpolating } \\
\text { BSPs }\end{array}$ & $0.65[0.44 ; 0.77]$ & $0.85[0.78 ; 0.94]$ \\
\hline
\end{tabular}

Both methods showed poor reconstruction at the apical $\mathrm{RV}$, where reconstructions completely fail to capture the topology of the EGMs. This is reflected by high RE values in this region. This is expected, as this area of the heart is closest to the region of broken electrodes on the 
tank. Overall there is little to no difference between the reconstructions when interpolated and removing broken BSPs. Across all cardiac sequences, there was little difference between the reconstruction accuracy of each method (Table 1), with no significant difference between $\mathrm{CC}(\mathrm{p}=0.20)$ or $\mathrm{RE}$ values $(\mathrm{p}=0.37)$.

\section{Discussion}

Our results show that inverse solutions are most affected by BSPs missing in regions of high potential gradient (centred over the heart). Furthermore, linear interpolation doesn't improve, and can further deteriorate reconstruction accuracy compared to simply removing electrodes. This is perhaps unsurprising, as previous studies conducted to optimise the number and arrangement of body surface electrodes [8,9], have defined body surface locations near the heart as containing the richest sources of relevant data. In these high gradient areas, it is likely that interpolation has inaccurately reconstructed missing BSPs (as in Fig. 1). As the inverse problem is already ill-posed, any further error introduced through interpolation would result in a less accurate inverse solution, compared to simply removing the electrodes, a result consistent with other studies $[10,11]$. Though an alternative interpolation scheme could be used, previous studies have shown it is very difficult for any interpolation method to accurately reconstruct missing BSPs in high gradient regions, and that the Barycentric method used here is one of the best for a low computational cost [11]. In addition, interpolation is accurate in regions of low potential gradients, where high density recording of BSPs is redundant. This means removing some of these BSPs doesn't affect the reconstruction accuracy, and thus produces similar EGMs to using interpolation (e.g. with the torso tank data).

What is perhaps interesting is that even when large amounts of seemingly important information on the body surface is removed, inverse solutions are still fairly accurate across the ventricles. In particular, the magnitudes of EGMS were more substantially affected than their topology ( $2 \%$ of EGMs with $\mathrm{CC}<0.95,26 \% \mathrm{RE}$ $>10 \%$ ), meaning activation pathways can be accurately delineated. This faithful reconstruction demonstrates the presence of considerable redundant information in the BSP measurements.

However, the results presented should be interpreted in light of the limitations inherent in the study. That is, redundant information found in simulated data may be important in the context of real potential measurements where geometric error is present. Furthermore, though experimental data were used, the presence of redundant information cannot be postulated from these results as the full set of potential measurements was not available on the tank. Further analysis of the data may reveal regions of low confidence on the ventricles when particular electrodes are missing on the body surface.

\section{Conclusion}

Broken or missing BSPs can reduce the accuracy of inverse reconstructions depending on their location and number. Linear interpolation to reconstruct lost BSPs does not improve, and in some cases further deteriorates reconstruction accuracy.

\section{Acknowledgements}

This study was funded by the Leducq Fondation and grant ANR-10-IAHU-04.

\section{References}

[1] Sapp JL, Dawoud F, Clements JC, Horacek B, Horácek BM. Inverse solution mapping of epicardial potentials: quantitative comparison with epicardial contact mapping. Circ Arrhythm Electrophysiol 2012;5(5): 1001-1009.

[2] Cochet H, Dubois R, Sacher F, Derval N, Sermesant M, Hocini M, Montaudon M, Haïssaguerre M, Maurent F, Jaïs, P. Cardiac arrythmias: Multimodal assessment integrating body surface ECG mapping into cardiac imaging. Radiology 2014;271(1):239-247.

[3] Ten Tusscher KHWJ. A Model for Human Ventricular Tissue. AJP Heart Circ Physiol 2003;286(4):H1573-89.

[4] Aras K, Good W, Tate J, Burton B, Brooks DH, Coll-Font J, Doessel O, Schulze W, Potyagaylo D, Wang L, van Dam P, MacLeod RS. Experimental Data and Geometric Analysis Repository - EDGAR J Electrocardiol. 2015 (in press)

[5] Barr RC, Ramsey M, Spach MS. Relating epicardial to body surface potential distributions by means of transfer coefficients based on geometry measurements. IEEE Trans. Biomed. Eng 1977:24(1);1-11.

[6] Wang Y, Rudy,Y. Application of the method of fundamental solutions to potential-based inverse electrocardiography. Ann Biomed Eng 2006:34(8);127288.

[8] Lux RL, Evans AK, Burgess MJ, Wyatt RF, Abildskov JA. Redundancy reduction for improved display and analysis of body surface potential maps. I. Spatial compression. Circulation Research, 1981:49(1);197-203.

[9] Hintermüller C, Fischer G, Seger M, Pfeifer B, Hanser F, Modre R, Tilg B. Multi-lead ecg electrode array for clinical application of electrocardiographic inverse problem. In Proc. Ann. Int. IEEE EMBS 2004:1;1941-4.

[10] Ghodrati A, Brooks DH, Macleod R. Methods of Solving Reduced Lead Systems for Inverse Electrocardiography. IEEE Trans Biomed Eng 2007:54(2);339-43.

[11] Burnes JE, Kaelber DC, Taccardi B, Lux RL, Ershler P. R., Rudy Y. A Field-Compatible Method for Interpolating Biopotentials. Ann Biomed Eng 1998;26(1):37-47.

Address for correspondence.

Laura Bear.

IHU-LIRYC, Hôpital Xavier Arnozan

Avenue du Haut Lévèque,

33600 Pessac, France. 